This manuscript was accepted and published by the Journal of Analytical and Applied Pyrolysis.

Publication data of the final, corrected work:

Várhegyi, G.: Aims and methods in non-isothermal reaction kinetics. J. Anal. Appl.

Pyrolysis 2007, 79, 278-288. doi: $10.1016 /$ j.jaap.2007.01.007

\title{
Aims and methods in non-isothermal reaction kinetics
}

\author{
Gábor Várhegyi \\ Institute of Materials and Environmental Chemistry, Chemical Research Center, Hungarian Academy of \\ Sciences, P.O. Box 17, Budapest, Hungary 1525. \\ Email: varhegyi.gabor@t-online.hu or gvarhegyi@gmail.com
}

\begin{abstract}
The majority of the works dealing with non-isothermal kinetics assumes only one kinetic differential equation, uses linearization techniques and do not check the fit between the simulated and the experimental data. It is not clear from the literature why we need kinetic evaluations at all in this field. Due to this controversial situation, the author has outlined his views on the aims and methods of the non-isothermal kinetics in this paper. Accordingly, the goal of the kinetic evaluation is to obtain better, more informative results from the experiments. If realistic models are used, numerous unknown parameters have to be determined during the evaluation. Since the most important errors of thermal analysis are not random, the laws of the mathematical statistics do not offer means to find the best set of model parameters. Nevertheless, the simultaneous evaluation of a series of thermoanalytical experiments by the method of least squares aims directly at the description of the sample behavior in a wide range of experimental conditions and helps the determination of a large number of unknown parameters. The outlined considerations are supported by examples from the work of the author and his coworkers. As a comparison, a statistical survey is given on those papers that were published in journals specialized for thermal analysis, thermochemistry and pyrolysis in 2006 and contained the term "kinetic" or "kinetics" in their titles.
\end{abstract}

\section{Introduction}

The subject of this paper is the evaluation of thermal analysis experiments by reaction kinetic methods. "Thermal analysis" itself has a broad, general definition: A sample is subjected to a $T(t)$ temperature program while we measure one or more quantities characteristic to the sample's behavior as a function of time or temperature. Though the official ICTAC definition, as published in 1979 [1], mentions only measurements as "function of temperature", isothermal experiments have always been present in the literature of the thermal analysis. Besides, almost all modern thermoanalytical equipments measure quantities as function of time. Virtually all sort of time-resolved pyrolysis experiments fall into this category. The actual examples discussed in the paper are based on the measurement of the sample mass (thermogravimetry, TG), heat flow rate (differential scanning calorimetry, DSC) and mass spectrometric intensities (in thermogravimetry - mass spectrometry, TG-MS). The number of scientific papers using thermal analysis methods can be estimated to be around 10000 yearly from the recent data of the Science Citation Index.

The term "kinetics" is used in a broad sense in thermal analysis. It covers the study and modeling of the rate(s) of changes of the measured quantities. The systems of the kinetic studies may vary from high 
purity inorganic substances undergoing physical phase transitions to the thermal decomposition of materials with extremely complex chemical composition. The term "non-isothermal" in the title is not restricted to linear $\mathrm{T}(\mathrm{t})$ functions; it includes all $T(t) \neq$ const heating programs.

The recent literature on the non-isothermal kinetics shows a rather mixed picture. As discussed in section 6 , the majority of the works in this field presents kinetic calculations without a reliable comparison of the resulting models to the experimental data. There are recent reviews in the field that do not emphasize the necessity of a model validation by checking the fit between the experiments and the data predicted from the model $[2,3]$. On the other hand, there are overviews that treat this point in details and give recommendations for the use of the method of least squares to obtain models that fit well the experiments $[4,5,6]$.

A similar controversy exists on the kinetic models, too. Though several papers have already emphasized that the complexity of the phenomena occurring during most non-isothermal experiments requires models built from more than one kinetic equation, the majority of the published non-isothermal kinetic analyses is based on oversimplified, one-reaction models.

The situation is illustrated in more details in Section 6, when a statistical survey is presented on papers that

(a) were published in 2006 in journals specialized for thermal analysis, thermochemistry and pyrolysis.

(b) and contained the term kinetic (kinetic, kinetics, thermokinetic, etc.) in their titles

There is a similar diversity in the aims of the kinetic evaluation, too. In a recent Mettler-Award lecture Vyazovkin expressed the opinion that the activation energies calculated from non-isothermal experiments are theoretically important quantities even if the given paper do not present a model [7]. On the other hand, the large number of questionable kinetic papers published regularly in this field has aroused skepticism on the meaning and value of non-isothermal kinetics. This skepticism was recently sharply expressed by Howell [8] in a paper dedicated to the memory of David Dollimore. He wrote: "The results of such studies have often formed the basis for the proposal of the 'mechanism' of reaction. This despite the fact that the reaction being observed is often unknown or is not a single process but rather several parallel or consecutive events. This latter is particularly true for 'variable temperature kinetics'. The utility/value of such exercises is marginal at best and contributes nothing to an understanding of the mechanism of any of the reactions involved."

Due to the controversial situation outlined above I would like to outline my views on the aims and methods of the non-isothermal kinetics in the present paper. These views were concluded from a work of more than three decades in this field. Examples from our work during these decades will be given as a support for the outlined views.

\section{Experimental}

The experiments quoted in this paper were carried out by the following apparatuses:

- $\quad$ Perkin Elmer DSC 2

- Perkin Elmer TGS-2

- Setaram DSC 111

- A TG-MS system built from a Perkin-Elmer TGS-2 thermobalance and a Hiden HAL quadrupole mass spectrometer

The description of the equipment and the procedures are given in the references cited in the treatment. A particular care was taken to avoid heat transfer problems, as discussed below, in section 3.3. 


\section{About the aims of the kinetic evaluation in thermal analysis}

\subsection{Even the qualitative interpretation of the experiments may need a kinetic background}

Every experimental technique needs some theoretical background. Without a theory, even the qualitative interpretation of the results may be problematic. This will be illustrated by a simple example. It is well known that the non-isothermal experimental curves in thermal analysis usually shift to higher temperatures with increasing heating rate. Frequently their shape does not change noticeably during this shift. Fig. 1 shows the behavior of a sample of ground willow wood. (See reference [9] for more details about the corresponding experiments.) Note that the DTG curves are expressed as $-\mathrm{dm} / \mathrm{dT}$ in Fig. 1 for a better visibility since the $-\mathrm{dm} / \mathrm{dt}$ curves have very different peak heights.

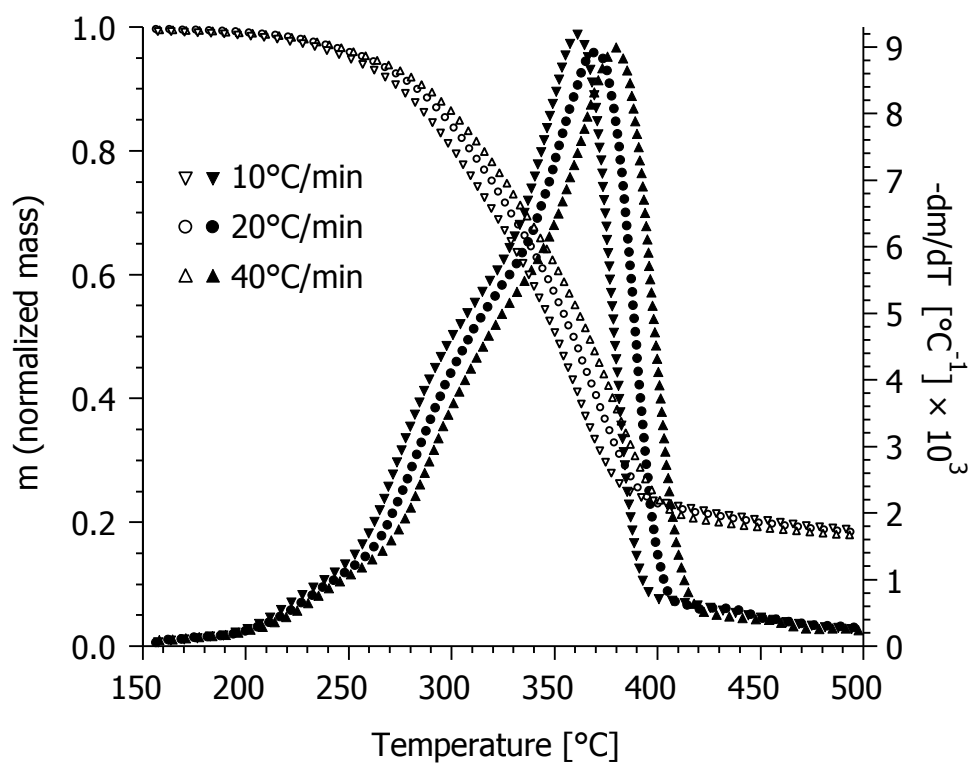

Fig. 1. The shift of the TG and DTG curves of a wood sample at increasing heating rates. (Here the DTG curves are expressed as $-\mathrm{dm} / \mathrm{dT}$ to improve visibility.)

We cannot interpret this phenomenon without a kinetic model. On the other hand, it is well known that the shift of the thermoanalytical curves can be derived mathematically from the following type of kinetic equations:

$$
\mathrm{d} \alpha / \mathrm{dt}=A \mathrm{e}^{-E / R T} \mathrm{f}(\alpha)
$$

where $\alpha$ is the reacted fraction of the given process, $A$ is the preexponential factor, $E$ is the activation energy, and $\mathrm{f}(\alpha)$ is a continuous function. (Here we use the IUPAC terminology for the activation energy: it is an empirical parameter characterizing the exponential temperature dependence of the rate coefficient [10].) The deduction immediately follows from the Coats - Redfern type of linearizations [11] and the corresponding formulae have been used for kinetic evaluation for almost 40 years [12]. Obviously, a wood is too complex material to be described by a singly kinetic differential equation. However, as an approximation, we can regard it to be composed from pseudocomponents, where a pseudocomponent is a fraction of reactive species that exhibit similar reactivity. A kinetic equation of type (1) is assumed for each pseudocomponent. The resulting mass loss rate curve is the weighted sum of the individual reaction rates:

$$
-\mathrm{dm} / \mathrm{dt}=\sum_{j=1}^{N_{\text {comp }}} c_{j} d \alpha_{j} / d t
$$

where $m$ is the normalized sample mass, $N_{\text {comp }}$ is the number of pseudocomponents and $c_{\mathrm{j}}$ is the normalized mass of volatiles formed from pseudocomponent $j$. An example is shown in Fig. 2 which is the result of the kinetic evaluation of a series of experiments [13]. We cannot assign a specific chemical 
component of a partial process unambiguously, since lignin, for example, decomposes in a wide range of temperature interval and, accordingly, contributes to the mass loss of all partial curves. Either we can employ the term "pseudocomponents" or we describe the situation as "partial curves dominated by ...", as it is done in Fig. 2. The phenomenon shown in Fig. 1 corresponds to the mathematical properties of this model in the usual range of its parameters.

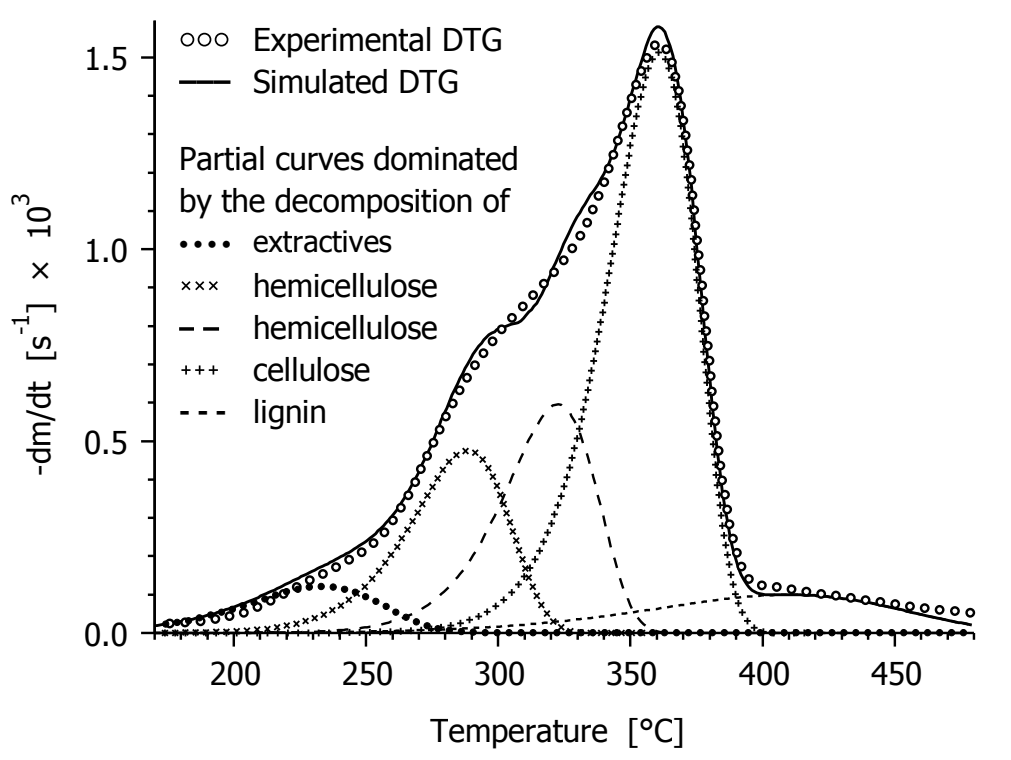

Fig. 2. The description of wood pyrolysis by a kinetic model of five pseudocomponents [13]. The model parameters were determined from a series of experiments; the present figure shows a DTG curve measured at linear heating with $10^{\circ} \mathrm{C} / \mathrm{min}$.

\subsection{What should we expect from a good kinetic model?}

The answer of this question depends obviously on the interest of the investigator and on the properties of the studied samples. Nevertheless, a few general criteria can obviously be listed:

- Description of the behavior of the samples in a wide range of experimental conditions

- Prediction of the behavior outside the domain of the given set of observations

- Characteristics that can reveal similarities and differences between the samples

- A deeper insight into the processes taking place ...

The ellipsis indicates that this list can probably be continued. It may be worth to have a look on the term "deeper insight" through a simple example. Fig. 3 shows examples for the numeric characteristics that can be read from the thermoanalytical curves without a kinetic evaluation. The notations $T_{3 \text { rd onset }}$ and $T_{1 \text { st offset }}$ indicates that more than one onset and offset values can be read from a complex thermoanalytical curve. The quantities shown in Fig. 3 are useful to compare experiments that were measured at identical experimental conditions. However, they do not reveal anything about the processes taking place. From this respect, the curve resolution shown in Fig. 2 is much more informative about the thermal behavior of this sample. 


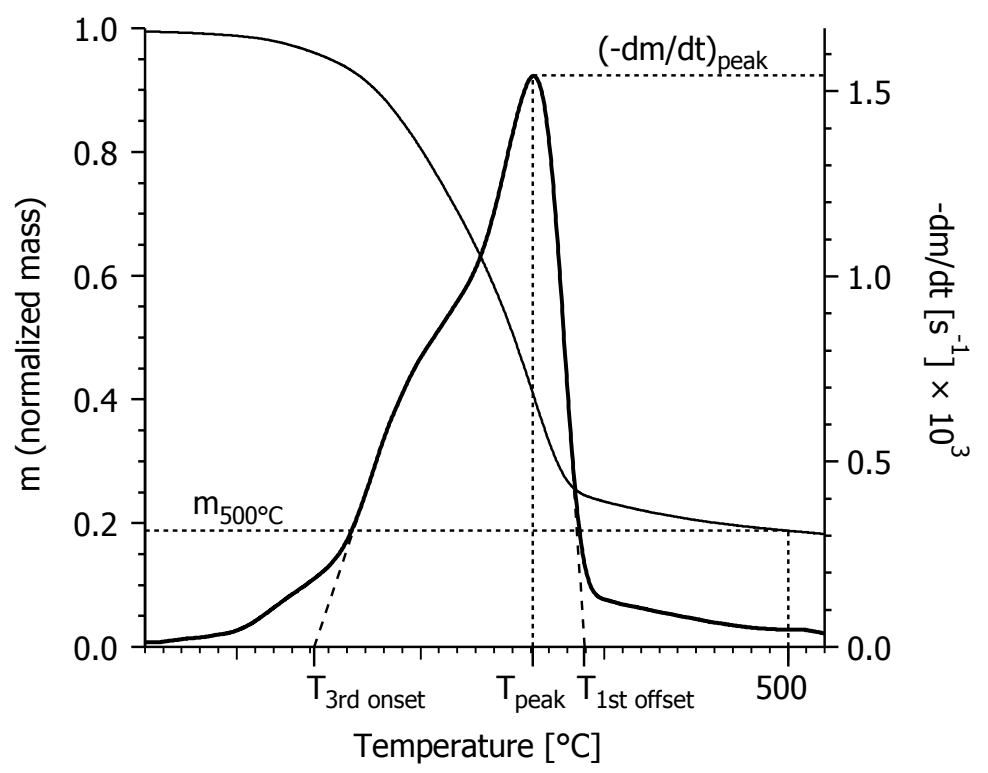

Fig. 3. Numerical characteristics that can be read from the experiment of Fig. 2 without a kinetic evaluation.

\subsection{The regime of kinetic control}

We assume in the treatment that the samples are under kinetic control during the experiments, meaning that the transport processes do not generate significant macroscopic inhomogeneities in the samples and the product formation is governed by chemical reactions. The reasons for this restriction are practical. A substantial transport control is usually an unnecessary complication in kinetic studies. It would be desirable to obtain information simultaneously on the transport processes and the reaction kinetics in many cases, but the given thermoanalytical equipments do not provide sufficient information for that purpose. The problem is illustrated by Fig. 4 that shows the combustion of a solid fuel. At low sample mass (bold line) the combustion occurs under kinetic control, in a temperature domain of ca. $130^{\circ} \mathrm{C}$. This curve is suitable for kinetic modeling [14,15]. If the sample mass is too high, the sample is ignited, and is consumed within seconds by an uncontrolled combustion, as shown by the thin line in Fig. 4. In this case the real temperatures in the sample are not known. Besides, most thermobalances cannot measure very sharp transitions correctly due to inertia, damping circuits or filters. It may be worth mentioning that Fig. 4 does not refer to a particularly reactive sample. When charcoals are heated in air, we observed significant self heating at $0.3 \mathrm{mg}$ and $25^{\circ} \mathrm{C} / \mathrm{min}$ [16], while the examination of wood in air required samples of around $0.4 \mathrm{mg}$ at $10^{\circ} \mathrm{C} / \mathrm{min}$ and $0.2 \mathrm{mg}$ at $20^{\circ} \mathrm{C} / \mathrm{min}$ [9]. (Note that the heat release rate is usually roughly proportional to the heating rate in the kinetic regime.) The above examples are observations in the same thermogravimetric apparatus (Perkin Elmer TGS-2) using a sample pan of $\varnothing 6$ $\mathrm{mm}$. 


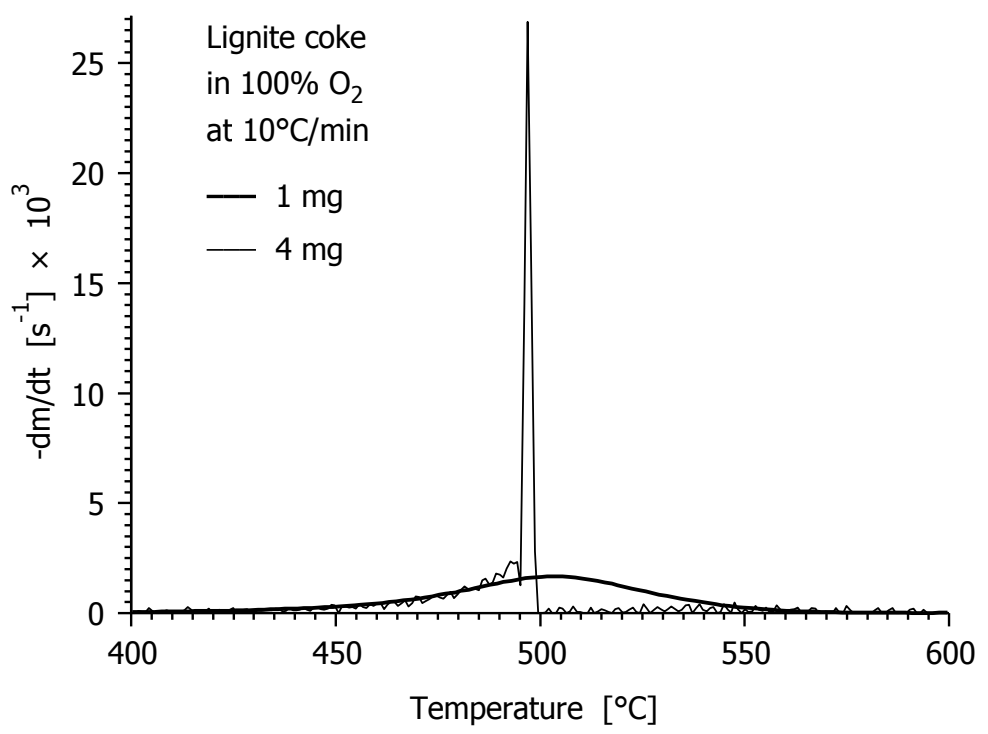

Fig 4. DTG curves of a solid fuel [14] in oxygen. Thin solid line: ignition due to a too high sample mass. Bold solid line: combustion under kinetic control.

\section{About the methods of kinetic evaluation}

\subsection{Collecting the necessary amount of kinetic information}

Most samples studied by thermal analysis are too complex to be described by only one chemical reaction. A more realistic model contains numerous parameters that should be determined during the kinetic evaluation process. The kinetic model shown in Fig. 2, for example, employs five first order reactions with 15 unknown parameters. (Each reaction has an $E$ and an $A$ parameter, and the weight factors of the pseudocomponents in Eq. (2) should also be determined.) Obviously one TG experiment cannot provide enough information for so many parameters. One should evaluate series of experiments. However, some care is needed in their planning. Fig. 1, for example, shows the strong similarities of the experiments carried out at linear $T(t)$ programs at different heating rates. Consequently, the adding more linear $T(t)$ experiments to the series shown there would only slightly increase the information content of the series. Other type of heating programs, however, may add further information, as shown in Fig. 5. (Obviously there are other ways, too, to increase the amount of information in a series of thermoanalytical curves.)
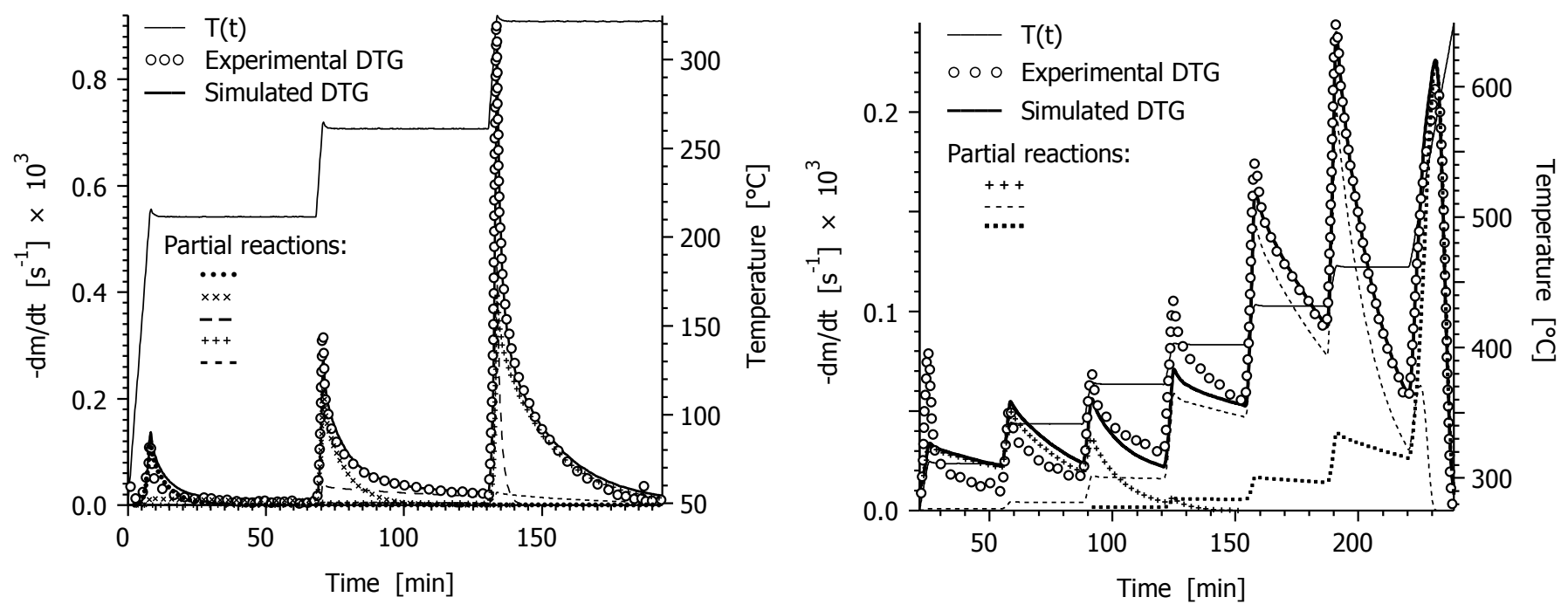

Fig. 5. Kinetic modeling of DTG curves at stepwise heating programs: (a) wood pyrolysis [13]; (b) charcoal combustion [16]. 


\subsection{The method of least squares as a practical tool for finding the unknown parameters}

The most important experimental errors of the thermal analysis are usually neither random nor independent. The most frequently occurring experimental error is the difference between the measured temperature and the actual temperature of the sample. Other typical non-random experimental errors are the baseline uncertainties. Even the noise of a thermogravimetric curve appears usually as irregular waves instead of a random scattering. Besides, the employed models are only approximate; we cannot expect them to describe perfectly our observations. In this way, we do not have data scattering randomly around some ideal, theoretical values. Consequently, the laws of the mathematical statistics cannot help us to find a "best" set for the unknown parameters. Lacking a statistical background, we have to look for another general criterion to find suitable parameters during the evaluation.

As outlined earlier, we expect a good model to describe the experimental data in a wide range of experimental conditions. If the particular research problem does not offer other, more specific requirements, we can use this general statement as a criterion to find good values for the unknown parameters. One should collect experimental data in a broad range of experimental conditions and evaluate them. If the term "describe the experimental data" means that the simulated and observed thermoanalytical curves are close to each other, then the straightforward way to ensure it is the application of the method of least squares to the whole set of experiments:

$$
S=\sum_{k=1}^{N_{\text {exp }}} \sum_{i=1}^{N_{k}} w_{\mathrm{i}, \mathrm{k}}\left[X_{\mathrm{k}}{ }^{\mathrm{obs}}\left(t_{\mathrm{i}}\right)-X_{\mathrm{k}}{ }^{\mathrm{calc}}\left(t_{\mathrm{i}}\right)\right]^{2} / N_{\mathrm{k}}^{2}=\min
$$

Here $S$ is the least squares sum to be minimized, subscript $k$ indicates the experiments differing in the experimental conditions. $N_{\exp }$ is the number of experiments evaluated simultaneously, $t_{i}$ denotes the time values in which the digitized values of the observations, $X_{\mathrm{k}}{ }^{\mathrm{obs}}\left(t_{\mathrm{i}}\right)$ were taken, and $N_{k}$ is the number of the $t_{i}$ points in a given experiment. $X_{\mathrm{k}}^{\text {calc }}$ represents the simulated counterparts of the observed curves obtained from the model at the given set of experimental parameters. Weight factors $w_{\mathrm{i}, \mathrm{k}}$ serve to emphasize the reliability or importance of the experiments or experimental points. $w_{\mathrm{i}, \mathrm{k}}$ can be set to 1 if TG data are evaluated, while the DSC and DTG curves are usually normalized by their maximum to unit height in a least squares sum $[17,18]$.

Since there is no statistical background behind Eq. (3) in the thermal analysis, its solutions do not have "maximum likelihood" properties. It is only a practical way to get simulated data close to their experimental counterparts. Accordingly, we do not have means to select unambiguously a "best" set of the parameters. One can be content finding only "good" parameters, where the term "good" refers to the satisfaction of the criteria listed in section 3.2.

According to the experience of the author and his coworkers, the method of the least squares, as formulated by Eq. (3), works well in non-isothermal kinetics and there is no need to replace it by methods requiring simpler computer programming or less computational time. Linearization techniques or "modelfree" approaches may be helpful to find initial values for the iterations involved in the method of least squares, but the goals outlined in section 3.2 are better fulfilled by a method aiming directly at the description of the experimental data in a wide range of experimental conditions. According to my experience, the easiest way to find initial values for the method of least squares is the reusing of parameters obtained in other, earlier studies. One can gradually change the preexponential factors till the simulated curves have peak maxima in the present domain of observation, then carry out a preliminary optimization by changing only a limited number of parameters. This procedure usually results in suitable initial parameters.

The various linearization techniques of the non-isothermal reaction kinetics are seldom suitable for a true least squares evaluation. As an example, let us have a look on the well-known Coats - Redfern linearization [11]:

$$
\ln \frac{g(\alpha)}{T^{2}} \cong \ln \frac{A R}{\beta E}-E / R T
$$


where $g(\alpha)$ is the integral of $1 / f(\alpha)$ and $\beta$ is the heating rate. In rarely occurring, ideal cases the left hand side can be regarded as an experimental quantity and Eq. 4 may be used in least squares methods. The conditions for it are obvious: the treatment should be restricted for models containing only one reaction step; this reaction should obey a variant of Eq. (1); the evaluation is restricted to experiments with linear heating programs; $f(\alpha)$ should not contain unknown kinetic parameters; and $\alpha$ should be directly calculated from the experimental data. The latest criterion means that the evaluated thermoanalytical peak or step should be preceded and followed by zero reaction rate sections (e.g. by horizontal sections on a TG curve). In this case $\alpha$ can be calculated as

$$
\alpha(t)=\frac{M(0)-M(t)}{M(0)-M(\infty)}
$$

where $M(t)$ is either the sample mass of a TG curve or the integral of a differential thermoanalytical curve and $M(0)$ and $M(\infty)$ are its values before the start and after the termination of the given reaction. There are organic substances whose non-isothermal pyrolysis can be described by a one-reaction model. However, the determination of $M(\infty)$ is problematic since the formed residues are slowly carbonizing further as the temperature increases. In the case of cellulose pyrolysis, for example, a round-robin study showed that $m(\infty)=M(\infty) / M(0)$ should be determined together with the kinetic parameters since the reading of an $M(\infty)$ value from the sloping end of the TG curve is ambiguous [19]. This excluded the possibility of using the Coats - Redfern method or any other linearization technique in the method of least squares.

Note that the definition of the method of least squares strictly refers to experimental and simulated data. If we hide some model parameters into $\mathrm{X}^{\text {obs }}$ (like $\mathrm{M}(0), \mathrm{M}(\infty)$ or a formal reaction order) or if we put an experimental value with its own uncertainties into $X^{\text {calc }}$ (like the temperature in Eq. 4) then the method will not be the method of least squares any more and it will not aim directly at a close match between the experimental and the simulated curves. Besides, the linearization techniques of the non-isothermal kinetics have particularly unfavorable error propagation properties. The left hand side of the Coats-Redfern linearization, for example, tends to $-\infty$ as $\alpha$ tends to zero.

\subsection{Can the kinetic parameters vary with experimental conditions?}

In an ideal case the answer for the above question would be "no". In real cases, however, the models seldom provide a perfect description of all phenomena occurring in the experiments and the experimental data do not scatter around unbiased mean values. The chemical reactions and the physical properties of a sample usually depend on the experimental conditions. The physical properties (transport properties, density, etc.) of a solid sample obviously change more or less with temperature. Concerning the chemistry, one could expect side reactions at the upper temperatures of the domain of experimental conditions. There are well documented examples of side reactions at lower temperatures, too. For example, Broido and Weinstein [20] have shown that cellulose undergoes through char forming reactions if it spends longer times below ca. $300^{\circ} \mathrm{C}$ while devolatilization dominates at higher temperatures. Accordingly, char forming side reactions arise in low heating rate cellulose experiments, while the same side reactions are negligible at higher heating rates. Burnham and Weese have shown similar effects with other materials, too $[21]$.

The systematic experimental errors also depend on the experimental conditions. A well known example is the difference between the actual and the measured sample temperature that strongly depend on the heating rate. (The highest reaction rate is usually roughly proportional to the heating rate; accordingly the rate of heat release or heat consumption is also roughly proportional to the heating rate.) Besides, there are non-statistical experimental errors that vary from experiment to experiment even if all conditions are identical. These are the various base line uncertainties that alter the whole of a curve (instead of producing a random scattering within a curve).

There are several ways to cope with the listed problems. A few possibilities will be outlined below. The citations given refer partly to our own experience with the indicated approaches, and partly to other workers in the field of biomass research. 
(1) One can neglect the above problems by evaluating all experiments with exactly the same parameters. From a mathematical point of view, this is the simplest way. From a physical point of view, a flexible model with many adjustable parameters frequently can approximate formally most of the systematic errors. We employed this approach in our first work on the least squares evaluation in nonisothermal thermal analysis [22] as well as in a few recent papers [23,24,16]. In the field of biomass research this approach was introduced by Caballero et al. [18] and Teng et al. [25].

(2) There is a possibility to include parameters for the description of the systematic errors. Some care is needed here, however, if we introduce too many parameters, the evaluation of the unknown parameters becomes a mathematically ill-defined problem. One can employ zero order, first order, etc. approximations for the systematic errors. In the former case the means of the errors are described by constant terms. For example, a $\delta T_{\mathrm{k}}$ parameters describes the mean of the systematic temperature error of experiment $k$. See references [9] and [13] for further details about this approach. It is worth noting that the only reason for using this zero-order approximation is its simplicity; we did not wish to increase highly the number of unknown parameters in the model. There are detailed models about the effect of the heat transfer problems on kinetics in the literature [26-28].

(3) One can allow a few, selected parameters to be slightly different in the different experiments and keep other parameters identical. Permitting a slight scattering for the preexponetial factors, for example, allows the peak position of the partial processes to vary to some extent with the heating rate or other experimental conditions in the linear $T(t)$ experiments. If the $E_{\mathrm{j}}$ parameters and the optional parameters of the $f(\alpha)$ functions have the same values in all experiments, the shape and the width of the partial peaks are only slightly influenced. This approach emphasizes that the same model is valid in the given range of experimental conditions with minor uncertainties on the position of the partial peaks. See papers $[14,29,30]$ for further details.

(4) Sometimes one can evaluate each experiment separately and observe the similarities/differences of the parameters obtained from the different experiments. This approach works well if the information content of an experiment is sufficient for the determination of all unknown parameters. An example was shown by Cabellero et al. [18] by employing two first order reactions for DTG curves that exhibited partial peaks without a high overlap. In more complex cases, however, this approach leads to mathematically illdefined cases [15]. Another possibility is to allow each experiment in a series to have its own set of parameters, and employ mathematical techniques to force the parameter sets close to each other [17]. The results of this procedure reflect that the given model describes only approximately the phenomena occurring during the experiments.

It is difficult to select the "best" from the approaches listed in this section; the properties of the experiments and the model together determine which method can give suitable results in the given situation.

\section{The steps of the author and his coworkers in the outlined directions}

\subsection{The method of least squares for one or more experiments with models of more than one partial process (1979)}

The present author has published a paper in 1979 on the evaluation of thermoanalytical curves [22] that appears to be the first in the literature of thermal analysis from the following aspects of view: 


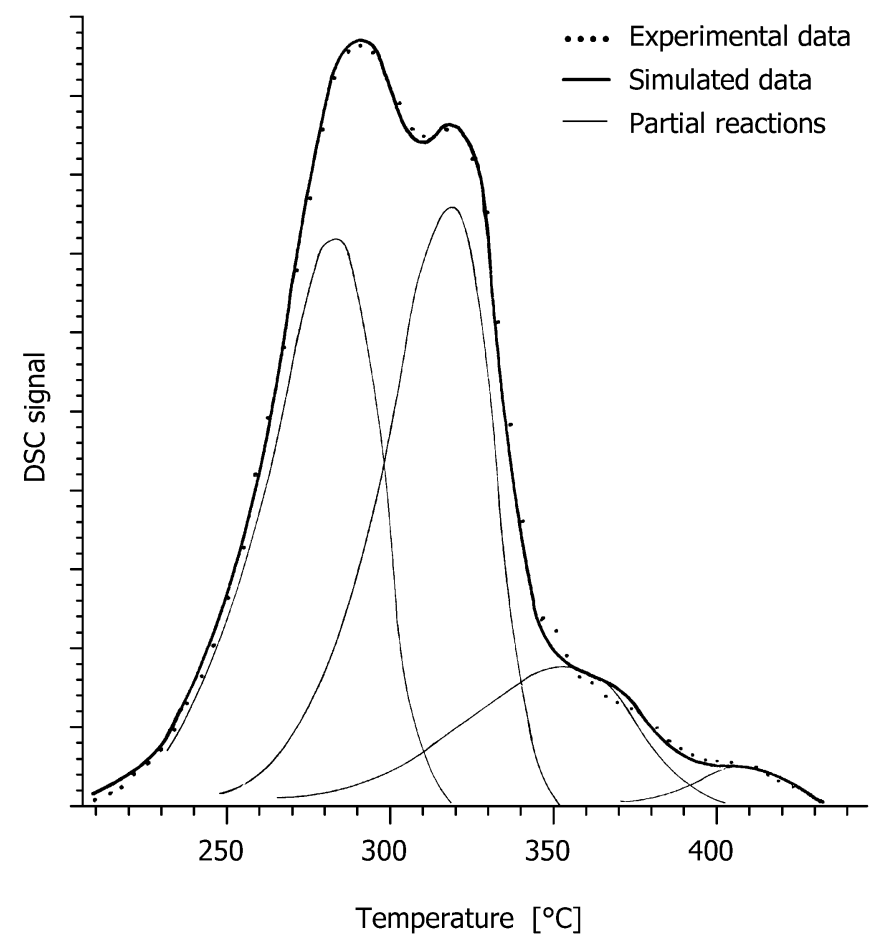

Fig 6. Kinetic evaluation of a DSC curve assuming 4 partial reactions. An application of the method of least squares for the thermal analysis of alloys in 1979 [31].

(i) This work assumed more than one partial process and aimed for the kinetic resolution of thermoanalytical signals of samples composed from more than one component. A subsequent publication [31] described its applications in studying phase transitions in metallic alloys by DSC. Fig. 6 displays an approximate resolution of a DSC curve to component curves by assuming $1^{\text {st }}$ order kinetics for the partial curves. This figure contains the scanned image of a nearly 30-year old, computer generated plot.

(ii) It was based on the method of least squares. It contained Eq. (3) of the present work without weight factors:

$$
S=\sum_{k=1}^{N_{\text {exp }}} \sum_{i=1}^{N_{k}}\left[X_{\mathrm{k}}{ }^{\mathrm{obs}}\left(t_{\mathrm{i}}\right)-X_{\mathrm{k}}{ }^{\mathrm{calc}}\left(t_{\mathrm{i}}\right)\right]^{2}
$$

where $S$ is the least squares sum to be minimized, subscript $k$ indicates the experiments differing in the experimental conditions and $N_{\exp }$ is the number of experiments evaluated simultaneously. The cases of $N_{\text {exp }}=1$ and $N_{\text {exp }}=2$ were considered and compared by numerical examples. The simulated counterparts of the experimental curves $X_{\mathrm{k}}{ }^{\text {calc }}(t)$ were obtained by the numerical solution of the kinetic equations of the partial processes. Though the non-linear method of least squares was already employed in many areas of the science in 1979, the kinetic evaluation in the non-isothermal thermal analysis was based usually on various linearization techniques or on approximate formulae that used only $1-3$ points from an experimental curve. One should mention, however, the pioneering work of Broido and Weinstein [20] who used an analog computer in their isothermal studies on cellulose decomposition. They used a complex model and they selected the best fitting solution by a visual comparison of the simulated and experimental curves on an oscilloscope. The lack of the non-linear method of least squares in the early literature of thermal analysis may be due to specific numerical problems caused by the non-isothermal $T(t)$ programs in the case of more than one partial reaction. Work [22] presented a computer algorithm to solve these problems. The algorithm and its later variants have been used by the author and his coworkers in 26 scientific publications from 1979 [31] till the present [16].

(iii) This work discussed the application of stepwise heating programs as a means to decrease the overlap of the partial reactions [22]. The simultaneous evaluation of linear and stepwise temperature programs was considered and tested on simulated data. (See Figure 7). 

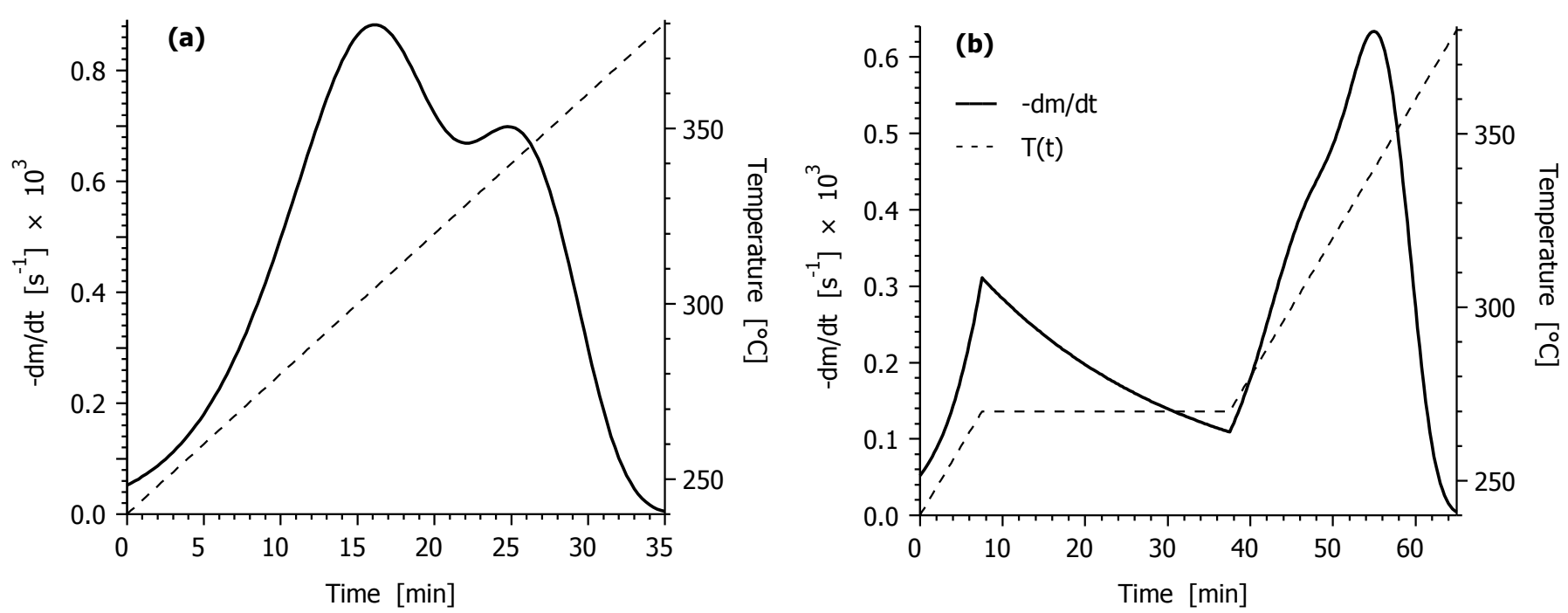

Fig. 7. Simulated curves at linear (a) and stepwise (b) heating programs assuming two overlapping first order reactions. They served as numerical examples for studying the simultaneous evaluation of experiments by the method of least squares in 1979 [22].

\subsection{Competitive, consecutive and parallel reactions (1989)}

In 1988 the above algorithm was extended for competitive, consecutive and parallel reactions, and was employed in biomass research [32]. In this work the unknown parameters of the employed models were not high, and the kinetic evaluation could be based on the separate evaluation of the experiments. Figure 8 shows results on catalyzed cellulose decomposition obtained by a model of two consecutive reactions. The decomposition of a biomass sample (sugar cane bagasse) was described by three parallel reactions. A few years later this description was supplemented by a low, wide partial reaction curve characteristic for lignin $[33,34]$ and the results become similar to the recent curve resolutions of biomass materials. (See e.g. Fig. 2 in the present paper for a recent result in this field.) Papers [33] and [34] also introduced the washing of the biomass samples by hot water or dilute acids as a pretreatment to decrease the overlap of the partial processes of the kinetic analysis.

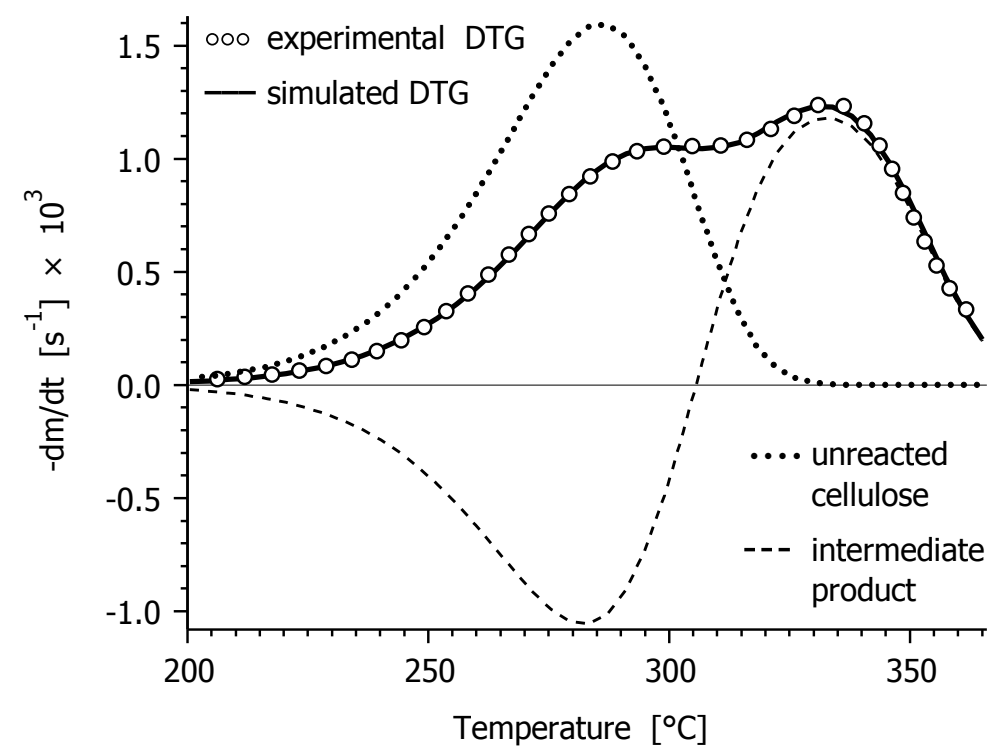

Fig. 8. $\mathrm{ZnCl}_{2}$-catalyzed decomposition of cellulose: A least squares kinetic evaluation in 1989 [32]. Successive reactions were assumed in the model: a dehydration reaction produced an intermediate (- - -) from the unreacted sample $(\bullet \bullet)$. (Since the vertical axis shows $-\mathrm{dm} / \mathrm{dt}$, the formation rates are below zero in the plot.) 


\subsection{Simultaneous evaluation of series of experiments by the method of least squares (1993- 1996)}

As outlined above, in sections $4.1-4.2$, if the model contains a larger number of unknown parameters, the evaluation should be based on series of experiments. In a work published in 1993 [17] we studied the thermal decomposition of cellulose in hermetically sealed, pressure-tight sample holders. Water vapor, which is a main product of the decomposition, proved to catalyze the reactions. The results were described by the following reaction scheme:

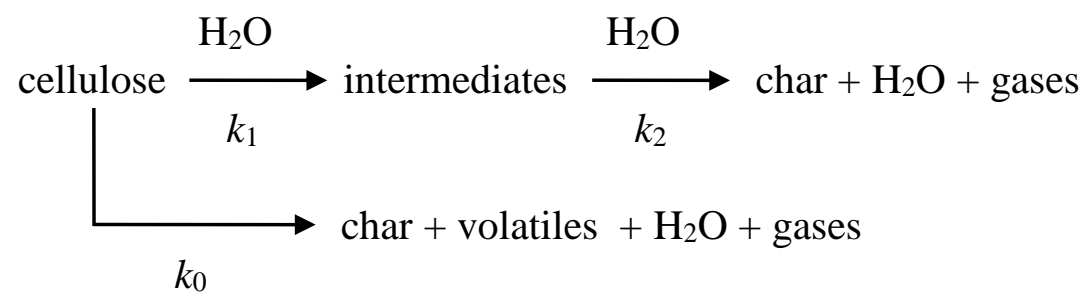

where $k_{0}, k_{1}$ and $k_{2}$ are rate constants with an Arrhenius-type dependence on the temperature. The unknown kinetic parameters of the model were the activation energies, preexponetial factors, and the water yields. The simulation of the DSC curves required the determination of the heats of the partial reactions, too; these values were added into the set of unknown parameters. The parameters associated to the lower branch of reaction scheme (7) were taken from literature sources; the rest were determined from a series of nine experiments by the method of least squares (Eq. (3)) using the considerations outlined in paragraph (4) of section 4.3. Fig. 9 shows the evaluation of nine DSC experiments differing in the conditions that determine the extent of water catalysis [17].

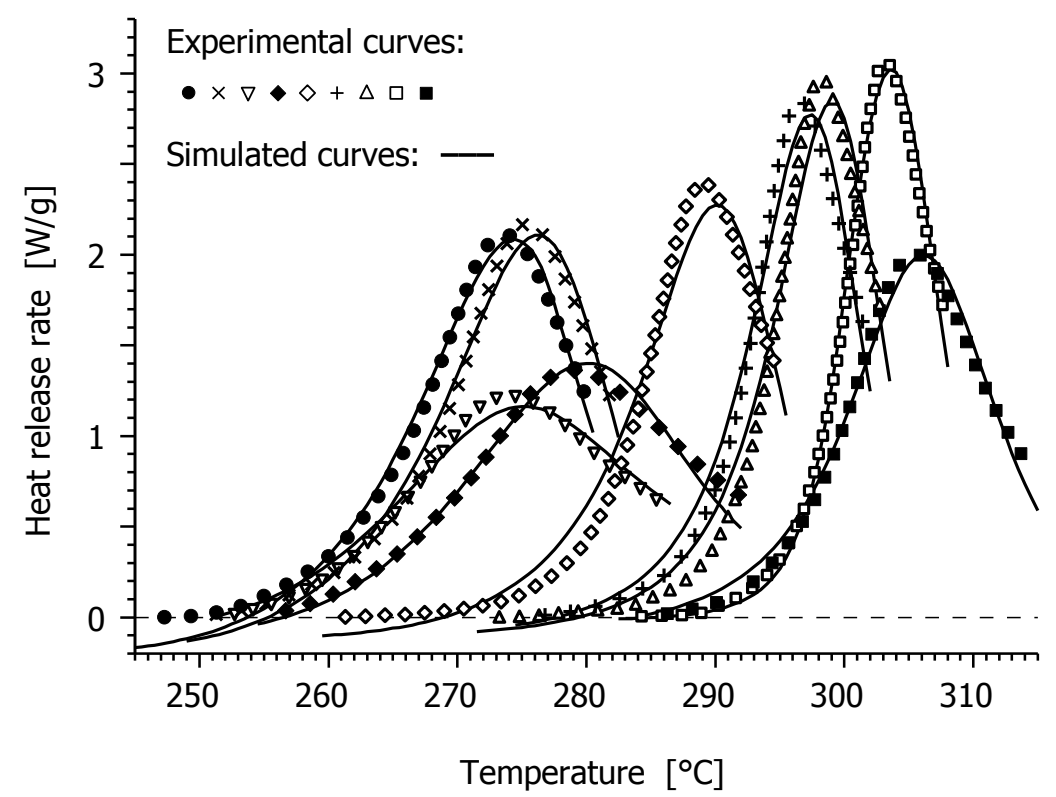

Fig. 9. Simultaneous evaluation of 9 DSC experiments in 1993 [17] by assuming a complex, autocatalytic mechanism. (See the text.)

Next year results were published on low and high temperature cellulose pyrolysis in open sample holders [29]. TG experiments were employed with $T(t)$ programs consisting of isothermal and nonisothermal sections. Four experiments with this type of stepwise $T(t)$ functions were evaluated simultaneously by models composed from two competitive and two - three consecutive reactions.

A paper published in 1996 [14] dealt with the combustion properties of coal chars (cokes). Series of experiments were evaluated by the method of least squares. Different temperature programs were used to increase the information content of the experimental data set, as shown in Fig. 10. Part of the samples was 
described by assuming two components in the char with different reactivities. Since the available theories of the char burn-off were deduced for strongly idealized cases and did not reflect the complexity of a real coke, we determined empirical $f(\alpha)$ reactivity functions in the evaluation by introducing a versatile empirical formula into Eq. (1). Two of the obtained empirical $f(\alpha)$ functions are shown in Fig. 11. Their shapes show a marked increase of the reaction surface at the first part of the burn-off. The curve represented by solid line started its sharp increase from a very low value reflecting either the opening of hardly accessible pores at the beginning of the combustion or an initial activation of the char surface. It may be worth noting that TG experiments cannot reveal reliable information on the beginning of the $f(\alpha)$ functions if the evaluation is based on linearizations instead of the general method of least squares. It is well known that the linear $T(t)$ experiments provide only ambiguous information on the shape of the $\mathrm{f}(\alpha)$ functions while the reaction kinetics based only on the isothermal parts of the experiments is loosing the information prior reaching the isothermal section.

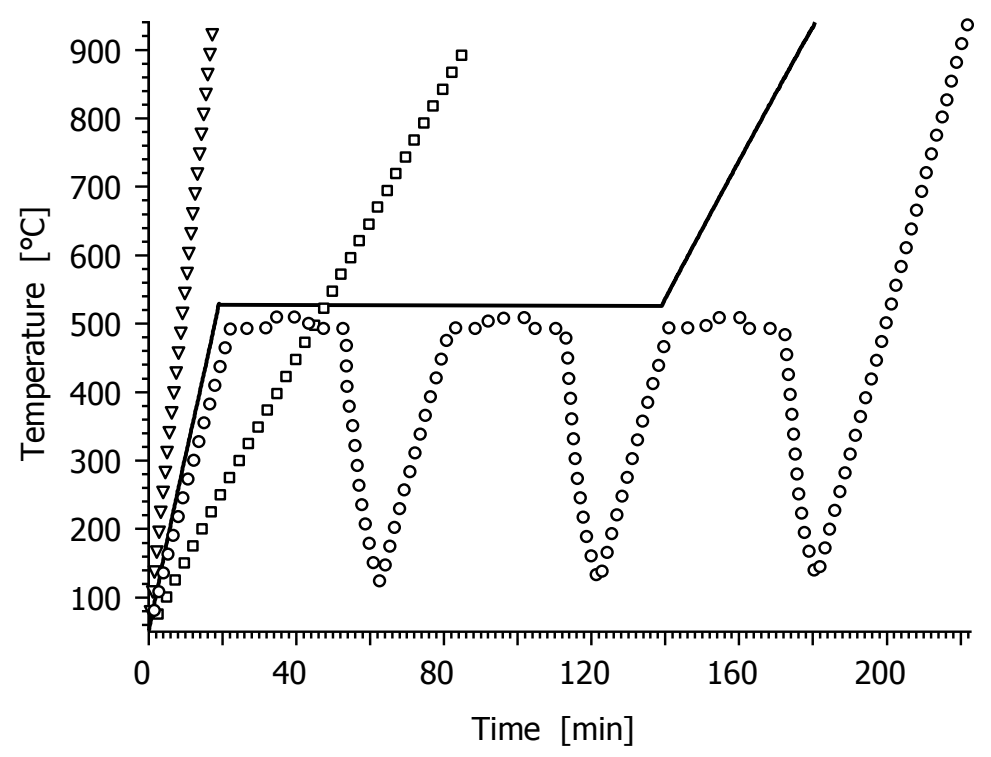

Fig. 10. Four temperature programs used to increase the information content of a series of experiments in 1996 [14].

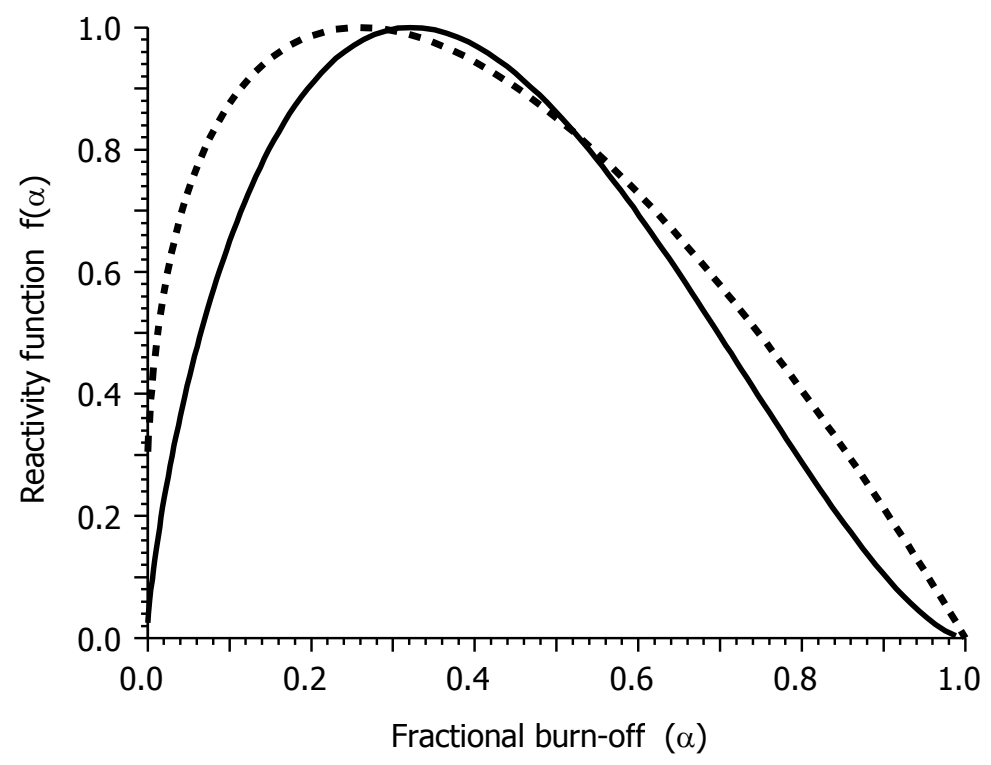

Fig. 11. Experimental reactivity functions for char burn-off reactions determined by the kinetic evaluation of a series of experiments in 1996 [14]. The two curves in the figure refer to two components with different reactivities in a Polish coke sample. 


\subsection{Our recent steps in the outlined directions}

The increasing availability of the computer power aided our work to proceed in two directions: we employed models that earlier could not be solved at general $T(t)$ functions; and, in other works, we increased the number of experiments evaluated simultaneously to determine the similarities and differences in larger data sets.

It is a frequent task to analyze samples of industrial importance that are not composed of well defined chemical compounds. If the properties of the reactive species follow some distributions in a sample, its behavior can be approximated by distributed activation energy models (DAEM). Burnham and Braun have published a high-level, detailed review on this field [4]. In our work we extended the applicability of the distributed activation energy models for the evaluation of series of experiments measured at general $\mathrm{T}(\mathrm{t})$ functions assuming more than one component or pseudocomponent [23]. In this way the model can reflect the real complexity of a coal, coke, charcoal or other carbonized solid fuel. As an example, Fig. 12 shows the methane formation from a devolatilizing charcoal. This process could be well described by one DAEM reaction at various heating programs.

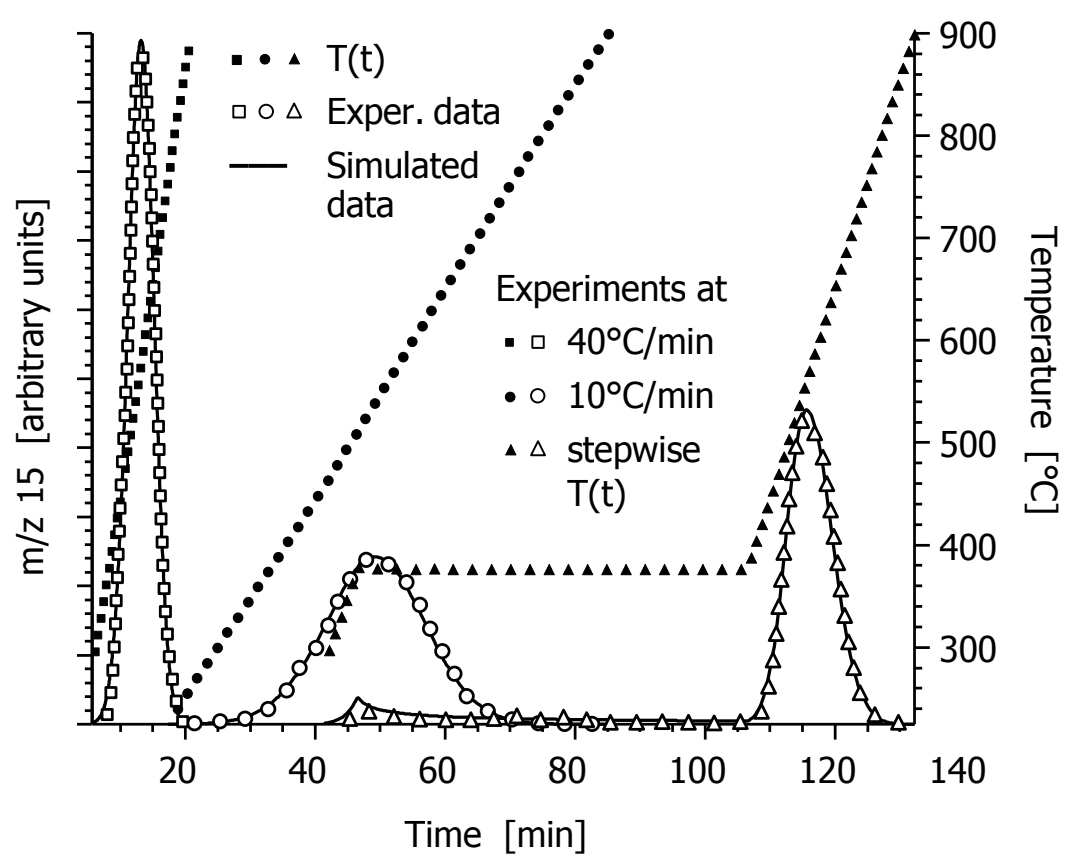

Fig. 12. Application of a distributed activation energy model (DAEM) for charcoal devolatilization [23]. A mass spectrometric intensity curve characteristic to methane evolution is shown at three different heating programs. (This is a subset of six experiments evaluated simultaneously by the method of least squares.)

In another work, the similarities and differences were searched in a larger set of experimental data by employing a common model and evaluating the experiments simultaneously by the method of least squares. Studying the wood products of an energy plantation, we evaluated a series of 12 experiments representing 2 grinding levels, 3 plant genera and 4 different heating programs [9]. Eq. (1) was employed with first order and $n$th order $f(\alpha)$ assuming $4-6$ pseudocomponents. In this model the wood species differed from each other only by the weight factors of the pseudocomponents in Eq. (2).

In a study of charcoal combustion, a series of 28 experiments on three charcoal samples were evaluated [16]. The experiments differed in their temperature programs, in the ambient gas composition and in the grinding of the samples. The model included a $1^{\text {st }}$ order devolatilization step and two burn-off reactions with empirical $f(\alpha)$ functions (similarly to our earlier work [14] shown in Fig. 11). Part of the calculations was based on the simultaneous evaluation of the 28 experiments with the determination of $42-84$ unknown parameters by the method of least squares. In other calculations each sample was evaluated separately estimating $27-36$ parameters from $8-12$ experiments by the method of least squares. The work resulted in an adequate description of the data over a wide range of experimental conditions within 
the kinetic regime. Characteristics of the combustion process were determined, including activation energy values characteristic for the temperature dependence of the burn-off; formal reaction orders characterizing the dependence on the oxygen content of the ambient; and $f(\alpha)$ functions describing the conversion dependence of the partial processes.

\section{A statistical survey on a sample from the latest literature in the field}

In this paragraph a brief survey is given of the papers that contain the term kinetic (kinetic, kinetics, thermokinetic, etc.) in their titles and were published in 2006 in journals specialized for thermal analysis, thermochemistry and pyrolysis: Journal of Thermal Analysis and Calorimetry; Thermochimica Acta; and Journal of Analytical and Applied Pyrolysis. In the 2006 volumes of these journals 77 titles contained the term kinetics/kinetic/thermokinetic. Of these papers 20 dealt with isothermal kinetics, 3 discussed theories without actual evaluations and 53 contained or included non-isothermal kinetic evaluation. This later group (53 papers) forms the basis of the present survey.

Eight papers $(15 \%)$ determined the unknown model parameters by the method of least squares. (Here the term "method of least squares" refer to non-linear least squares procedures, as defined by Eq. (3)). Another 7 papers (13\%) determined the model parameters by other means, but still checked and presented the fit between the experimental and the simulated data. I would like to emphasize that the comparison of the simulated and the experimental data is an inevitable step in the model validation; we cannot accept the validity of a given model without this step. Unfortunately, the remaining 38 papers $(72 \%)$ did not fulfill this basic criterion.

19 papers (36\%) published correlation coefficient values and several other works presented linearization plots without providing quantitative measures on the linearity. However, the linearization methods employed in these studies are not based on the comparison of the experimental data to quantities simulated from the model. Accordingly, there is no direct relationship between the correlation coefficients (or the linearity of the plots) and the actual fit of the model to the experimental data.

The majority of the papers described the processes by a single kinetic equation. Sometimes a kinetic equation is employed only for a section of the experimental curves without expressing how the corresponding $\alpha$ values are determined. (More precisely, how the $\mathrm{M}(0)$ and $\mathrm{M}(\infty)$ quantities are chosen for Eq. (5).) 30 papers contained the term "isoconversion" and 14 used the term "model-free" (with or without a dash between the words). Taking into account the common subset of these groups, and omitting one paper that used such methods only to obtain initial guesses for true least squares calculations [35], 39 papers (74\%) remain that reported evaluations by these methods. Both the "isoconversion" and the "model-free" approaches assume the validity of the following kinetic equation:

$$
\mathrm{d} \alpha / \mathrm{dt}=A \mathrm{e}^{-E(\alpha) / R T} f(\alpha)
$$

where $E(\alpha)$ and $f(\alpha)$ are continuous empirical functions. Several papers were based entirely on Eq. (8) while other works used it only as an alternative analysis of the data. Obviously an empirical $E(\alpha)$ function determined by these types of evaluations may be theoretically very interesting if (and only if) there is only one rate determining process in the given system. However, this is a relatively rare situation in the thermoanalytical studies. Besides, the validity of Eq. (8) should be proven even if a simple process is studied. After the determination of the $E(\alpha)$ and $f(\alpha)$ functions, one should solve equation (8) at the given heating programs of the study and compare the simulated curves to the experiments. There are standard computer procedures both for the representation of tabular $E(\alpha)$ and $f(\alpha)$ data by mathematical formulas and for the numerical solution of Eq. (8) for any $E(\alpha)$ and $f(\alpha)$. This part of the model validation was missing in the majority of the 39 papers employing Eq. (8) in the present sample. Only four papers in this group compared the solutions of Eq. (8) to the experiments. They selected $f(\alpha)$ models from the literature and used the mean values of the empirical $\mathrm{E}(\alpha)$ functions for the simulation. 


\section{Conclusions}

This paper assumes that the main goal of the kinetic evaluation of the thermal analysis experiments is to obtain better, more informative results from the experiments. Even the qualitative interpretation of the experiments may need a kinetic background. Besides, we usually expect that a kinetic modeling should provide

- Description of the behavior of the samples in a wide range of experimental conditions;

- Characteristics that can reveal similarities and differences between the samples

- A deeper insight into the processes occurring during the experiments.

If realistic models are used, numerous unknown parameters have to be determined during the evaluation. Since the most important errors of the thermal analysis are not random, the laws of the mathematical statistics and the method of the maximum likelihood do not offer means to find the best set of model parameters. Nevertheless, the simultaneous evaluation of a series of thermoanalytical experiments by the method of least squares aims directly at the description of the sample behavior in a wide range of experimental conditions and helps the determination of a large number of unknown parameters. The various linearization techniques of the non-isothermal reaction kinetics are seldom suitable for this purpose, since they cannot describe complex reaction mechanisms and they are limited to linear $T(t)$ programs.

The outlined considerations were discussed mainly on examples from the work of the author and his coworkers in the past three decades. The earlier example in the treatment was a paper of 1979 [22] that dealt with the application of the non-linear method of least squares for more than one experiment and more than one partial reaction at arbitrary $T(t)$ temperature programs. Parallel to the development of the computing techniques, the evaluation has been extended to more complex reaction mechanisms and to larger series of experiments. In our recent works we evaluate simultaneously groups of $8-28$ experiments that differ in the temperature programs and in other experimental conditions [16]. The models included parallel reactions where the partial reactions were approximated by first order, power-law [9], and general empirical $f(\alpha)$ functions [14,16] as well as distributed activation energy models [23]. We also employed reaction schemes composed from consecutive and competitive reactions [17,29].

The outlined considerations and examples were compared to the present state of the field by a simple statistical survey of the latest publications in the field of non-isothermal kinetics. The sample for this statistics included those papers that were published in 2006 in journals specialized for thermal analysis, thermochemistry and pyrolysis and contained the term kinetic in their titles. Serious problems were pointed out, including the frequent use of oversimplified, one-reaction models and the lack of the comparison between the model and the experimental data in the majority of the papers.

\section{Acknowledgements}

The author is grateful for the Hungarian National Research Fund (grants T37705 and T37704) and to Drs. Michael J. Antal, Jr, Emma Jakab, Erika Mészáros, William S. Mok, Piroska Szabó, Tamás Székely and Ferenc Till for their kind help in the work outlined.

\section{NOMENCLATURE}

$\alpha \quad$ reacted fraction of a sample or of a pseudocomponent

$A \quad$ pre-exponential factor $\left(\mathrm{s}^{-1}\right)$

$\beta \quad$ heating rate $(\mathrm{K} / \mathrm{s})$

$c \quad$ normalized mass of volatiles formed from a pseudocomponent

$E$ activation energy (an empirical parameter characterizing the exponential temperature dependence of the rate coefficient [10], $\mathrm{kJ} / \mathrm{mol}$ )

$f(\alpha) \quad$ a function expressing the dependence of the reaction rate on the conversion

$m \quad$ normalized sample mass (dimensionless) 
$M \quad$ sample mass (g or $\mathrm{mg}$ )

$N_{\text {comp }} \quad$ number of pseudocomponents

$N_{\text {exp }} \quad$ number of experiments evaluated simultaneously

$N_{\mathrm{k}} \quad$ number of evaluated data on the $k$ th experimental curve

$R \quad$ gas constant $\left(8.3143 \times 10^{-3} \mathrm{~kJ} \mathrm{~mol}^{-1} \mathrm{~K}^{-1}\right)$

$S \quad$ least squares sum

$t \quad$ time (s)

$T$ temperature $\left({ }^{\circ} \mathrm{C}, \mathrm{K}\right)$

$X^{\text {obs }}(t), X^{\text {calc }}(t)$ an experimental quantity and its simulated counterpart

Subscripts:

i digitized point on an experimental curve

$\mathrm{j}$ pseudocomponent

$\mathrm{k} \quad$ experiment

\section{References}

1. R. C. Mackenzie: Nomenclature in thermal-analysis. Part 4. Thermochim. Acta 28 (1979) 1-6.

2. A. Khawam and D. R. Flanagan Basics and applications of solid-state kinetics: A pharmaceutical perspective. J. Pharmaceutical Sci. 95 (2006) 472-498.

3. S. Vyazovkin: Thermal analysis. Anal. Chem. 76 (2004) 3299-3311.

4. A. K. Burnham and R. L. Braun: Global kinetic analysis of complex materials. Energy Fuels 13 (1999) 122.

5. J.A. Conesa, A. Marcilla, J.A. Caballero and R. Font: Comments on the validity and utility of the different methods for kinetic analysis of thermogravimetric data. J. Anal. Appl. Pyrolysis 58-59 (2001) 617-633.

6. J. A. Caballero and J. A. Conesa: Mathematical considerations for non-isothermal kinetics in thermal decomposition. J. Anal. Appl. Pyrolysis 73 (2005) 85-100.

7. S. Vyazovkin: Model-free kinetics. Staying free of multiplying entities without necessity. J. Thermal Anal. Calorimetry 83 (2006) 45-51.

8. B. A. Howell: Utility of kinetic analysis in the determination of reaction mechanism. J. Thermal Anal. Calorimetry 85 (2006) 165-167.

9. E. Mészáros, G. Várhegyi, E. Jakab and B. Marosvölgyi: Thermogravimetric and reaction kinetic analysis of biomass samples from an energy plantation. Energy Fuels, 18 (2004) 497-507.

10. IUPAC Compendium of Chemical Terminology, $2^{\text {nd }}$ Edition, International Union of Pure and Applied Chemistry, 1997. Available on the internet: http://www.iupac.org/publications/compendium/index.html.

11. A. W. Coats and J. P. Redfern: Kinetics parameters from thermogravimetric data. Nature, 201 (1964) 6869.

12. H. E. Kissinger: Reaction kinetics in differential thermal analysis. Anal. Chem. 29 (1957) 1702-1706.

13. G. Várhegyi, E. Mészáros and E. Jakab: Advanced computational methods for the characterization of biomass samples by thermogravimetric analysis. In Biomass for Energy, Industry and Climate Protection, Proceedings of the 2nd World Biomass Conference, Edited by W. P. M. Van Swaaij et al., pp. 902-905, ETA and WIP, Florence, 2004.

14. G. Várhegyi, P. Szabó, E. Jakab, F. Till and J-R. Richard: Mathematical modeling of char reactivity in Ar$\mathrm{O}_{2}$ and $\mathrm{CO}_{2}-\mathrm{O}_{2}$ mixtures. Energy Fuels, 10 (1996) 1208-1214

15. G. Várhegyi, P. Szabó, E. Jakab and F. Till: Least squares criteria for the kinetic evaluation of thermoanalytical experiments. Examples from a char reactivity study. J. Anal. Appl. Pyrolysis, 57 (2001) 203-222. 
16. G. Várhegyi, E. Mészáros, M. J. Antal, Jr., J. Bourke and E. Jakab: Combustion kinetics of corncob charcoal and partially demineralized corncob charcoal in the kinetic regime. Ind. Eng. Chem. Res. 45 (2006) 4962-4970.

17. G. Várhegyi, P. Szabó, W. S. L. Mok and M. J. Antal, Jr.: Kinetics of the thermal decomposition of cellulose in sealed vessels at elevated pressures. Effects of the presence of water on the reaction mechanism. J. Anal. Appl. Pyrolysis, 26 (1993) 159-174.

18. J. A. Caballero, J. A. Conesa, R. Font and A. Marcilla: Pyrolysis kinetics of almond shells and olive stones considering their organic fraction. J. Anal. Appl. Pyrolysis, 42 (1997) 159-175.

19. M. Grønli, M. J. Antal, Jr., G. Várhegyi: A round-robin study of cellulose pyrolysis kinetics by thermogravimetry. Ind. Eng. Chem. Res. 38 (1999) 2238-2244.

20. A. Broido and M. Weinstein: Kinetics of solid-phase cellulose pyrolysis. In Proceedings of the 3rd International Conference on Thermal Analysis, H. G. Wiedemann (Ed), Birkhauser Verlag: Basel, 1971; pp 285-296.

21. A. K. Burnham and R. K. Weese: Kinetics of thermal degradation of explosive binders Viton A, Estane, and Kel-F. Thermochim. Acta 426 (2005) 85-92.

22. G. Várhegyi: Kinetic evaluation of non-isothermal thermoanalytical curves in the case of independent reactions. Thermochim. Acta, 28 (1979) 367-376.

23. G. Várhegyi, P. Szabó, M. J. Antal, Jr.: Kinetics of charcoal devolatilization. Energy Fuels, 16 (2002) 724-731.

24. C. J. Gómez, G. Várhegyi, L. Puigjaner: Slow pyrolysis of woody residues and an herbaceous biomass crop: A kinetic study. Ind. Eng. Chem. Res. 44 (2005) 6650-6660.

25. H. Teng, H. C. Lin and J. A. Ho: Thermogravimetric analysis on global mass loss kinetics of rice hull pyrolysis. Ind. Eng. Chem. Res. 1997, 36, 3974-3977.

26. R. Narayan, M. J. Antal, Jr., Thermal lag, fusion and compensation effect during biomass pyrolysis. Energy Fuels, 35 (1996) 1711-1721.

27. H. Bockhorn, A. Hornung, U. Hornung, P. Jakobströer, Modelling of isothermal and dynamic pyrolysis of plastics considering non-homogeneous temperature distribution and detailed degradation mechanism. $J$. Anal. Apl. Pyrolysis, 49 (1999) 53-74.

28. H. Bockhorn, A. Hornung, U. Hornung, Mechanism and kinetics of thermal decomposition of plastics from isothermal and dynamic measurements. J. Anal. Appl. Pyrolysis, 50 (1999) 77-101.

29. G. Várhegyi, E. Jakab, M. J. Antal, Jr.: Is the Broido - Shafizadeh model for cellulose pyrolysis true? Energy Fuels, 8 (1994) 1345-1352.

30. H. Teng and Y-C. Wei: Thermogravimetric Studies on the Kinetics of Rice Hull Pyrolysis and the Influence of Water Treatment. Ind. Eng. Chem. Res., 37 (1998), 3806 -3811.

31. G. Várhegyi, G. Groma and M. Lengyel: DSC examination of alloys. Thermochim. Acta, 30 (1979) 311317.

32. G. Várhegyi, M. J. Antal, Jr., T. Székely, P. Szabó: Kinetics of the thermal decomposition of cellulose, hemicellulose and sugar cane bagasse. Energy Fuels, 3 (1989) 329-335.

33. G. Várhegyi, P. Szabó, M. J. Antal, Jr.: Reaction kinetics of the thermal decomposition of cellulose and hemicellulose in biomass materials. In Advances in Thermochemical Biomass Conversion (Ed. by A. V. Bridgwater), Volume 2, Blackie Academic and Professional, London, 1994, pp. 760-771.

34. G. Várhegyi, P. Szabó, M. J. Antal, Jr.: Kinetics of the thermal decomposition of cellulose under the experimental conditions of thermal analysis. Theoretical extrapolations to high heating rates. Biomass Bioenergy, 7 (1994) 69-74.

35. H. Geßwein and J.R. Binder: Thermokinetic study of the oxidation of $\mathrm{ZrAl}_{3}$ powders. Thermochim. Acta 444 (2006) 6-12. 